\title{
A facile regio- and stereoselective synthesis of trans-ethyl 5-aroyl-4- aryl-2-[(arylsulfonyl)methyl]-4,5-dihydrofuran-3-carboxylates
}

\author{
Palani Sokkan Harikrishnan, ${ }^{a}$ Stephen Michael Rajesh, ${ }^{a}$ Abdulrahman I. Almansour, ${ }^{b}$ and \\ Subbu Perumal ${ }^{\mathrm{a}, *}$ \\ ${ }^{a}$ Department of Organic Chemistry, School of Chemistry, Madurai Kamaraj University, Madurai \\ 625 021, India \\ ${ }^{b}$ Department of Chemistry, College of Science, King Saud University, PO Box 2455, Riyadh \\ 11451, Saudi Arabia \\ Email:subbu.perum@gmail.com
}

\begin{abstract}
A library of novel trans-ethyl 5-aroyl-4-aryl-2-[(arylsulfonyl)methyl]-4,5-dihydrofuran-3carboxylates were synthesized regio- and stereoselectively in good yields by a three-component domino reaction of ethyl-3-oxo-4-(arylsulfonyl)butanoates, aromatic aldehydes and $\mathrm{N}$ phenacylpyridinium bromide in presence of triethylamine in acetonitrile under heating. This transformation generates $\mathrm{C}-\mathrm{O}, \mathrm{C}-\mathrm{C}$ and $\mathrm{C}=\mathrm{C}$ bonds and presumably proceeds via an $\alpha, \beta$ unsaturated ketosulfonyl ester generation/Michael addition/intramolecular cyclisation domino sequence.
\end{abstract}

Keywords: Domino reaction, ethyl-3-oxo-4-(arylsulfonyl)butanoates, aromatic aldehyde, dihydrofuran

\section{Introduction}

Multicomponent reactions (MCRs) have emerged as an important synthetic strategy for generating complex scaffolds of structural diversity from simple molecules. In this protocol, three or more reactants are assembled together in a one pot operation to form a new product comprising substantial portions of all the components. ${ }^{1-3}$ MCRs, besides generating complex structures, provide operational simplicity and synthetic efficiency over conventional linear multistep reactions. The most useful MCRs have additional advantages of selectivity, synthetic convergence, atom-economy and green credentials. ${ }^{4-9}$ Further, MCRs are perfectly amenable for automation to generate combinatorial libraries of biologically relevant heterocyclic scaffolds and hence play a pivotal role in lead identification and drug development programmes. 
Furan and di-/tetrahydrofuran sub-structures are prevalent in diverse classes of natural products and biologically active heterocycles, besides serving as versatile building blocks in organic synthesis ${ }^{10-12}$ and several synthetically derived sulfones also show interesting biological activities (Figure 1). For instance, dapsone $\mathbf{1}$ is an important drug currently used for the treatment of leprosy in conjunction with other drugs. ${ }^{13}$ 1-(4-Trifluoromethyl)-phenyl-2(phenylsulfonyl)ethanone 2 inhibits of $11 \beta$-hydroxysteroid dehydrogenase activity, ${ }^{14}$ whilst substituted (E)-(2-chloro-2-(phenylsulfonyl)vinyl)benzenes 3 show antiplasmodial activity. ${ }^{15}$ Sulfones appended to furan moiety also show important biological activities. For example, functionalized vinyl dihydrofuryl sulfones $\mathbf{4}$ and tetrahydrofuryl sulfones $\mathbf{5}$ show antiprotozoal ${ }^{16}$ and antimycobacterial activity ${ }^{17}$ respectively. Consequently, in the present work, we report the regio- and stereoselective synthesis of highly functionalized biologically relevant dihydrofurans bearing arylsulfonyl moiety 6.<smiles>Nc1ccc(S(=O)(=O)c2ccc(N)cc2)cc1</smiles>

1

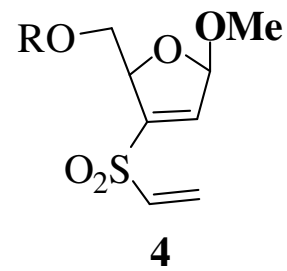<smiles>O=C(CS(=O)(=O)c1ccccc1)c1ccc(C(F)(F)F)cc1</smiles>

2<smiles>[R]c1ccc(C=C(Cl)S(=O)c2ccccc2)cc1</smiles>

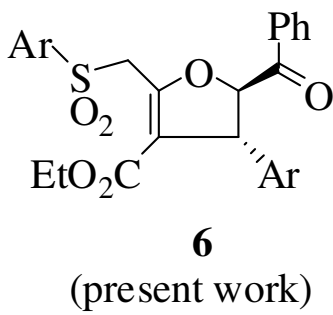

\section{Figure 1}

Previously reported synthesis of dihydrofuran derivatives include the reactions of sulfur ylide with $\alpha$-ylidene- $\beta$-diketones via [4+1] annulation reaction, ${ }^{18,19}$ one-step cyclization of $\beta$ ketosulfides of benzothiazole and aldehydes in ionic liquids, ${ }^{20}$ reaction of $\beta$ ketopolyfluoroalkane sulfones with aldehydes, ${ }^{21}$ reactions of 1,3-dicarbonyl compounds with appropriate olefins, ${ }^{22-24}$ or $\alpha$-bromonitroalkenes. ${ }^{25}$ Cyclization as well as ring enlargement reaction are mostly used for the construction of dihydrofurans. ${ }^{26-29}$ The reaction of pyridinium ylides with $\alpha, \beta$-unsaturated carbonyl systems, ${ }^{30-33}$ is one of the most familiar and frequently used synthetic methods for the construction of dihydrofurans, the ylides, in turn, can be easily prepared by deprotonation of the $\alpha$-halogenocarbonyl compounds. Present study constitutes a part of our ongoing research program launched on the exploration of tandem/domino/sequential multi-component reactions for the assembly of novel heterocycles. ${ }^{34-38}$ 


\section{Results and Discussion}

In the present investigation, a library of novel trans-ethyl 5-aroyl-4-aryl-2[(arylsulfonyl)methyl]-4,5-dihydrofuran-3-carboxylates $\mathbf{1 0}$ were synthesized regio- and stereoselectively in good yields by a three-component domino reaction of ethyl-3-oxo-4(arylsulfonyl)butanoates 7, aromatic aldehydes $\mathbf{8}$ and $N$-phenacylpyridinium bromide $\mathbf{9}$ in the presence of triethylamine in acetonitrile under heating. The base- and sovent-screen for the model reaction leading to $\mathbf{1 0 k}$ was studied and the results are presented in Table 1 . The model reaction was carried out with ethyl 3-oxo-4-(4-chlorophenylsulfonyl)butanoate 7n (1 mmol), $p$ chlorobenzaldehyde $\mathbf{8 n}(1 \mathrm{mmol})$ and $N$-phenacylpyridinium bromide 9 (1 mmol) in presence of base $(2.5 \mathrm{mmol})$ in various solvents under heating to reflux. It is found that $\mathrm{Et}_{3} \mathrm{~N}$ is more efficient for this transformation than either DBU or piperidine. A maximum yield of 10k (82\%) was obtained, when $\mathrm{Et}_{3} \mathrm{~N}$ was employed as the base and $\mathrm{CH}_{3} \mathrm{CN}$ as the solvent.

Table 1. Base- and solvent-screen for the synthesis of $\mathbf{1 0 k}$

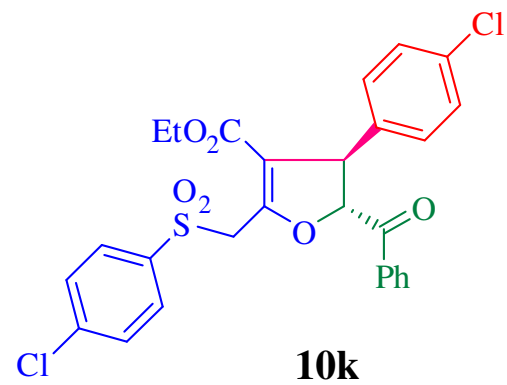

\begin{tabular}{ccccc}
\hline Entry & Base & Solvent & $\begin{array}{c}\text { Reaction } \\
\text { time }(\mathrm{h})\end{array}$ & $\begin{array}{c}\text { Yield of } \\
10 \mathrm{k}(\%)\end{array}$ \\
\hline 1 & $\mathrm{NH}_{4} \mathrm{OAc}$ & $\mathrm{CH}_{3} \mathrm{CN}$ & 4 & 45 \\
2 & $\mathrm{~K}_{2} \mathrm{CO}_{3}$ & $\mathrm{CH}_{3} \mathrm{CN}$ & 4 & 60 \\
3 & $\mathrm{Et}_{3} \mathrm{~N}$ & $\mathrm{CH}_{3} \mathrm{CN}$ & 4 & 82 \\
4 & $\mathrm{DBU}$ & $\mathrm{CH}_{3} \mathrm{CN}$ & 4 & 52 \\
5 & $\mathrm{Et}_{3} \mathrm{~N}$ & $\mathrm{CH}_{2} \mathrm{Cl}_{2}$ & 4 & 30 \\
6 & $\mathrm{Et}_{3} \mathrm{~N}$ & $\mathrm{MeOH}$ & 4 & 55 \\
7 & $\mathrm{Et}_{3} \mathrm{~N}$ & $\mathrm{DMF}$ & 4 & 65 \\
8 & $\mathrm{Et}_{3} \mathrm{~N}$ & $\mathrm{THF}$ & 4 & 68 \\
9 & $\mathrm{Piperidine}$ & $\mathrm{THF}$ & 4 & 48 \\
\hline
\end{tabular}


This optimized procedure was used for preparing a series of dihydrofuran derivatives employing substituted ethyl-3-oxo-4-(arylsulfonyl)butanoates, $N$-phenacylpyridinium bromide and aromatic aldehydes (Scheme 1. Table 2). Typically, the reaction was carried out by refluxing the reaction mixture of ethyl-3-oxo-4-(arylsulfonyl)butanoates 7 (1 mmol), aromatic aldehyde 8 $(1 \mathrm{mmol})$ and $\mathrm{N}$-phenacylpyridinium bromide $9(1 \mathrm{mmol})$ in presence of $\mathrm{Et}_{3} \mathrm{~N}$ (2.5 mmol) in

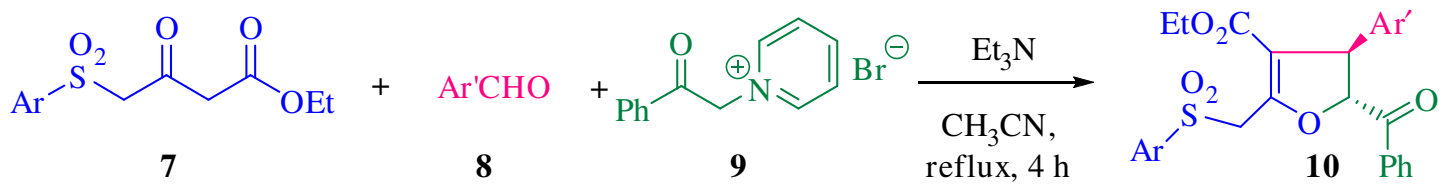

Scheme 1. Synthesis of trans-ethyl-5-aroyl-4-aryl-2-[(arylsulfonyl)methyl]- 4,5-dihydrofuran-3carboxylates $\mathbf{1 0}$.

Table 2. Yield and m.p. of $\mathbf{1 0}$

\begin{tabular}{cccccc}
\hline Entry & Comp. & $\mathrm{Ar}$ & $\mathrm{Ar}$ & $\mathrm{M} \cdot \mathrm{p}\left({ }^{\circ} \mathrm{C}\right)$ & $\begin{array}{c}\text { Yield of 10 } \\
(\%)\end{array}$ \\
\hline 1 & $\mathbf{1 0 a}$ & $p-\mathrm{MeC}_{6} \mathrm{H}_{4}$ & $\mathrm{C}_{6} \mathrm{H}_{5}$ & $126-127$ & 70 \\
2 & $\mathbf{1 0 b}$ & $p-\mathrm{MeC}_{6} \mathrm{H}_{4}$ & $p-\mathrm{MeC}_{6} \mathrm{H}_{4}$ & $112-113$ & 75 \\
3 & $\mathbf{1 0 c}$ & $p-\mathrm{MeC}_{6} \mathrm{H}_{4}$ & $p-\mathrm{ClC}_{6} \mathrm{H}_{4}$ & $148-149$ & 72 \\
4 & $\mathbf{1 0 d}$ & $p-\mathrm{MeC}_{6} \mathrm{H}_{4}$ & $p-\mathrm{O}_{2} \mathrm{NC}_{6} \mathrm{H}_{4}$ & $137-138$ & 71 \\
5 & $\mathbf{1 0 e}$ & $p-\mathrm{MeC}_{6} \mathrm{H}_{4}$ & $o-\mathrm{MeOC}_{6} \mathrm{H}_{4}$ & $115-116$ & 74 \\
6 & $\mathbf{1 0 f}$ & $p-\mathrm{MeC}_{6} \mathrm{H}_{4}$ & $o-\mathrm{BrC}_{6} \mathrm{H}_{4}$ & $132-133$ & 70 \\
7 & $\mathbf{1 0 g}$ & $p-\mathrm{MeC}_{6} \mathrm{H}_{4}$ & $m-\mathrm{FC}_{6} \mathrm{H}_{3}$ & $118-119$ & 77 \\
8 & $\mathbf{1 0 h}$ & $p-\mathrm{MeC}_{6} \mathrm{H}_{4}$ & $o, p-\mathrm{Cl}_{2} \mathrm{C}_{6} \mathrm{H}_{4}$ & $123-124$ & 75 \\
9 & $\mathbf{1 0}$ & $p-\mathrm{ClC}_{6} \mathrm{H}_{4}$ & $p-\mathrm{MeC}_{6} \mathrm{H}_{4}$ & $104-105$ & 80 \\
10 & $\mathbf{1 0 j}$ & $p-\mathrm{ClC}_{6} \mathrm{H}_{4}$ & $p-\mathrm{Pr}^{i} \mathrm{C}_{6} \mathrm{H}_{4}$ & $127-128$ & 72 \\
11 & $\mathbf{1 0 k}$ & $p-\mathrm{ClC}_{6} \mathrm{H}_{4}$ & $p-\mathrm{ClC}_{6} \mathrm{H}_{4}$ & $139-140$ & 82 \\
12 & $\mathbf{1 0 1}$ & $p-\mathrm{ClC}_{6} \mathrm{H}_{4}$ & $p-\mathrm{MeOC}_{6} \mathrm{H}_{4}$ & $120-121$ & 73 \\
13 & $\mathbf{1 0 m}$ & $p-\mathrm{ClC}_{6} \mathrm{H}_{4}$ & $o-\mathrm{BrC}_{6} \mathrm{H}_{4}$ & $128-129$ & 75 \\
14 & $\mathbf{1 0 n}$ & $p-\mathrm{ClC}_{6} \mathrm{H}_{4}$ & $m-\mathrm{BrC}_{6} \mathrm{H}_{3}$ & $130-131$ & 77 \\
15 & $\mathbf{1 0 0}$ & $p-\mathrm{ClC}_{6} \mathrm{H}_{4}$ & $m-\mathrm{FC}_{6} \mathrm{H}_{3}$ & $115-116$ & 72 \\
16 & $\mathbf{1 0 p}$ & $p-\mathrm{ClC}_{6} \mathrm{H}_{4}$ & $o, p-\mathrm{Cl}_{2} \mathrm{C}_{6} \mathrm{H}_{4}$ & $139-140$ & 70 \\
\hline
\end{tabular}

$\mathrm{CH}_{3} \mathrm{CN}$. After the completion of the reaction (TLC), the solvent was removed in a rotary evaporator and the residue was purified by column chromatography using ethyl acetate-pet.ether $(1: 4 \mathrm{v} / \mathrm{v})$ to give trans-ethyl 5-aroyl-4-aryl-2-[(arylsulfonyl)methyl]-4,5-dihydrofuran-3carboxylates $\mathbf{1 0}$ in good yields (70-82\%). 
The structure of the trans-4,5-dihydrofurans is in accord with elemental analyses and ${ }^{1} \mathrm{H},{ }^{13} \mathrm{C}$ and 2D NMR spectroscopic data as illustrated for a representative example, 10n (Figure 2). In the ${ }^{1} \mathrm{H}$ NMR spectrum of $\mathbf{1 0 n}, \mathrm{H}-4$ appearing as a doublet at $4.52 \mathrm{ppm}(J 5.2 \mathrm{~Hz})$ shows a H,HCOSY correlation with $\mathrm{H}-5$ appearing as a doublet at $5.69 \mathrm{ppm}(J 5.2 \mathrm{~Hz})$. The $J$ value shows that these $\mathrm{H}-4$ and $\mathrm{H}-5$ are in trans relationship. The $\mathrm{H}-4$ shows a $\mathrm{C}, \mathrm{H}-\mathrm{COSY}$ correlation with the carbon signal at $50.2 \mathrm{ppm}$ and also shows HMBCs with the C-5, C-3, C-2', C-1' ipso, C-2 and ketocarbonyl at 89.5, 112.4, 129.1, 139.8, 156.9 and 192.0 ppm respectively (Figures 2 and 3).

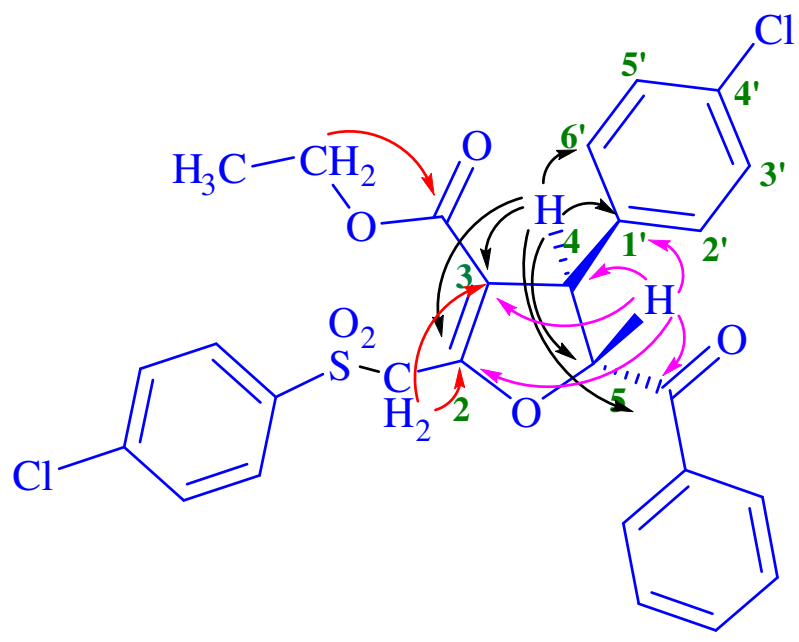

Figure 2. Selected HMBCs of 10n.

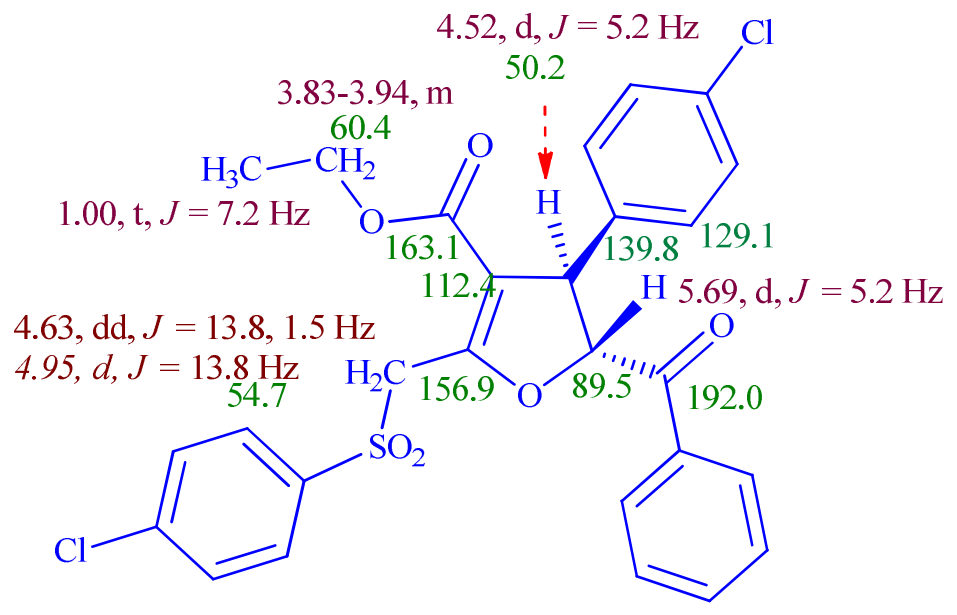

Figure 3. Selected NMR data of 10n.

The H-5 shows a C,H-COSY correlation with the carbon signal at $89.5 \mathrm{ppm}$ and shows HMBCs with C-4, C-3, C-1' and C-2 at 50.2, 112.4, 139.8 and 156.9 ppm respectively. The diastereotopic methylene hydrogens attached to the sulfonyl group give a doublet at $4.95 \mathrm{ppm}(\mathrm{J}$ $13.8 \mathrm{~Hz})$ and a doublet of doublets at $4.63 \mathrm{ppm}(J 13.8,1.5 \mathrm{~Hz})$ and these hydrogens show $\mathrm{C}, \mathrm{H}-$ 
COSY correlations with the carbon signal at $54.7 \mathrm{ppm}$ and HMBCs with C-2 and C-3 carbons. Ester methylene hydrogens appeared as a multiplet in the region of 3.86-3.94 ppm and methyl hydrogens appeared as a triplet at $1.00 \mathrm{ppm}(\mathrm{J} 7.2 \mathrm{~Hz})$. The structure of 10n deduced from NMR spectroscopic data is in good accord with that determined from the X-ray crystallographic studies on a single crystal of $\mathbf{1 0} \mathbf{n}^{39}$ (Figure 4).

This transformation leading to the formation of dihydrofurans (Scheme 2) is presumably triggered by an initial regioselective condensation of ketosulfonyl ester $\mathbf{7}$ with aromatic aldehyde 8 affording 11. This regioselectivity might probably arise from the steric hindrance posed by the bulky arylsulfonyl moiety to the condensation of the aromatic aldehyde with the methylene flanked by the sulfonyl and keto groups, which is likely to impede the formation of 11'. Subsequent Michael addition of the pyridinium ylide 12 to the acceptor $\mathbf{1 1}$ furnishes the enolate 13, which undergoes intramolecular cyclisation by the displacement of pyridine to afford exclusively the regioisomer $\mathbf{1 0 .}$

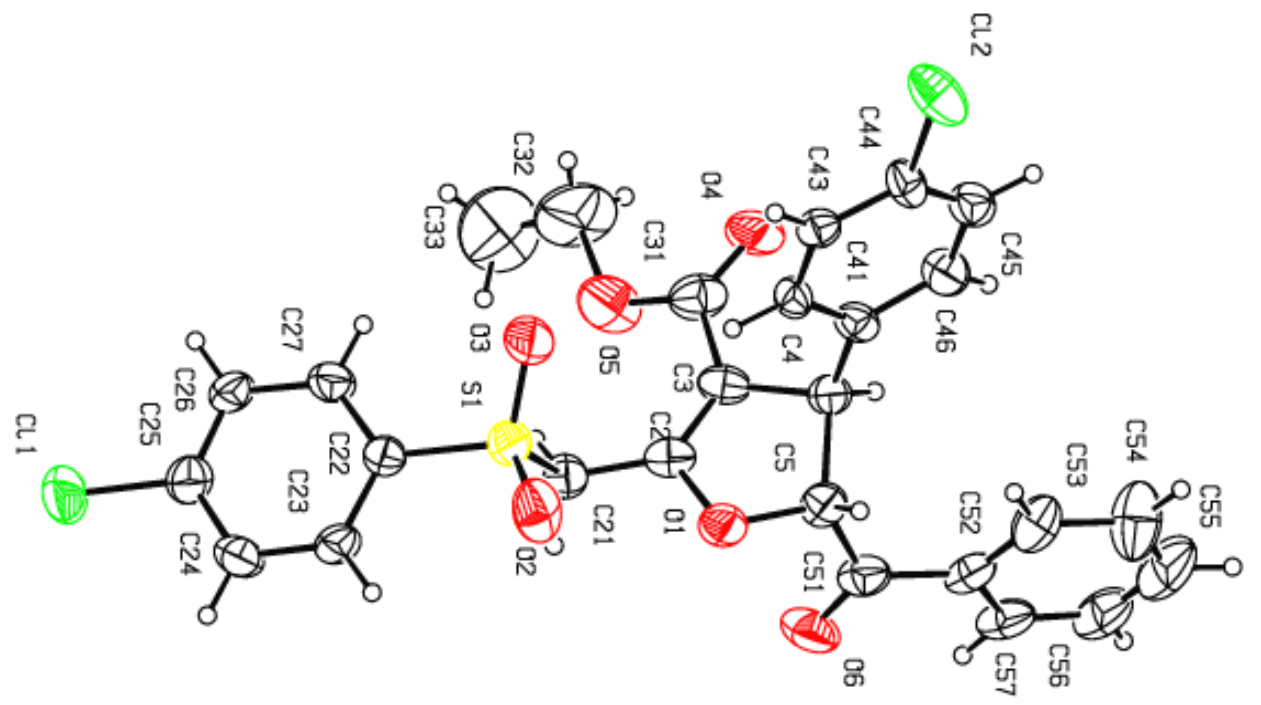

Figure 4. ORTEP diagram of 10n. 


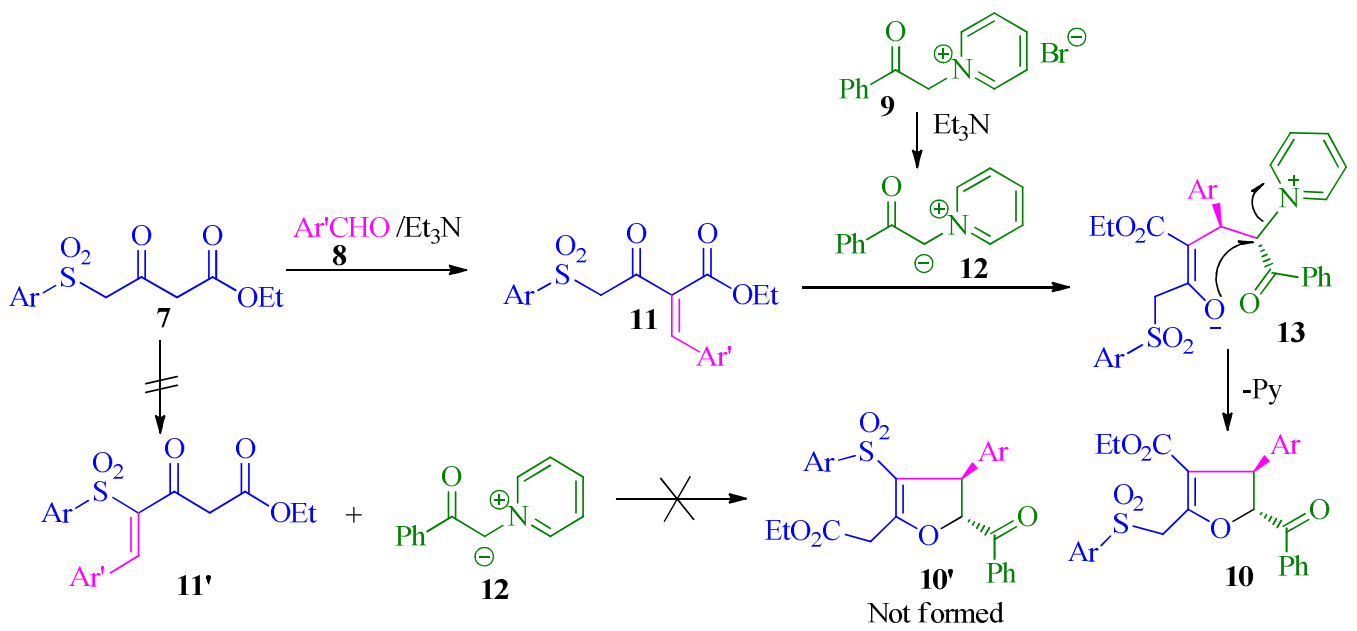

Scheme 2. Plausible mechanism for the formation of trans-ethyl 5-aroyl-4-aryl-2-[(arylsulfonyl)methyl]4,5-dihydrofuran-3-carboxylates $\mathbf{1 0}$.

\section{Conclusions}

In conclusion, the present work describes the synthesis of a series of novel trans-ethyl 5-aroyl-4aryl-2-[(arylsulfonyl)methyl]-4,5-dihydrofuran-3-carboxylates in a regio- and stereoselective manner in good yields via three-component domino reactions of ethyl-3-oxo-4(arylsulfonyl)butanoates, aromatic aldehydes and $\mathrm{N}$-phenacylpyridinium bromides in presence of triethylamine in acetonitrile under heating. This transformation generates $\mathrm{C}-\mathrm{O}, \mathrm{C}-\mathrm{C}$ and $\mathrm{C}=\mathrm{C}$ bonds and presumably proceeds via an $\alpha, \beta$-unsaturated ketosulfonyl ester generation/Michael addition/intramolecular cyclisation domino sequence.

\section{Experimental Section}

General. Melting points were measured in open capillary tubes and are uncorrected. The ${ }^{1} \mathrm{H}$ NMR, ${ }^{13} \mathrm{C}$ NMR, DEPT, H,H-COSY, C,H-COSY and HMBC spectra were recorded on a Bruker (Avance) $300 \mathrm{MHz}$ NMR instrument using TMS as internal standard and $\mathrm{CDCl}_{3}$ as solvent. Standard Bruker software was used throughout. Chemical shifts are given in parts per million $(\delta$ scale) and the coupling constants are given in Hertz. Silica gel-G plates (Merck) were used for TLC analysis with a mixture of pet. ether $\left(60-80{ }^{\circ} \mathrm{C}\right)$ and ethyl acetate as eluent. Elemental analyses were performed on a Perkin Elmer 2400 Series II Elemental CHNS analyzer.

\section{General synthesis of trans-ethyl 5-aroyl-4-aryl-2-[(arylsulfonyl)methyl]-4,5-dihydro-furan-} 3-carboxylates 10. A mixture of ethyl 3-oxo-4-(arylsulfonyl)butanoate 7 (1 mmol), aromatic aldehyde $8(1 \mathrm{mmol})$ and $\mathrm{N}$-phenacylpyridinium bromide $9(1 \mathrm{mmol})$ in the presence of $\mathrm{Et}_{3} \mathrm{~N}$ 
( $2.5 \mathrm{mmol}$ ) in $\mathrm{CH}_{3} \mathrm{CN}$ was heated to reflux for $4 \mathrm{~h}$. After completion of the reaction (TLC), the solvent was removed in a rotavapor and was purified by flash column chromatography with pet. ether-ethyl acetate $(4: 1 \mathrm{v} / \mathrm{v})$ mixture to afford pale yellow solid (10a-t). Spectroscopic data for all the compounds are given below.

trans-Ethyl5-benzoyl-4-phenyl-2-((p-methylphenylsulfonyl)methyl)-4,5-dihydrofuran-3carboxylate (10a). Pale yellow solid, yield 70\%, mp $126-127{ }^{\circ} \mathrm{C} .{ }^{1} \mathrm{H}$ NMR $\left(300 \mathrm{MHz}, \mathrm{CDCl}_{3}\right)$ $\delta_{\mathrm{H}}: 0.97\left(\mathrm{t}, 3 \mathrm{H}, J 7.2 \mathrm{~Hz}, \mathrm{CH}_{3}\right), 2.41\left(\mathrm{~s}, 3 \mathrm{H}, \mathrm{CH}_{3}\right)$ ) 3.82- $3.94\left(\mathrm{~m}, 2 \mathrm{H}, \mathrm{CH}_{2}\right), 4.51(\mathrm{~d}, 1 \mathrm{H}, J 5.1$ $\mathrm{Hz}), 4.55$ (d, 1H, J 13.8 Hz), 5.01 (d, 1H, J $13.8 \mathrm{~Hz}$, ), 5.44 (d. 1H, J 5.1 Hz), 7.22-7.38 (m, 7H, Ar-H), 7.45 (t, 2H, J 7.1 Hz, Ar-H), 7.61 (t, 1H, J 7.1 Hz, Ar-H), 7.28 (d, 2H, J 7.1 Hz, Ar-H), $7.86(\mathrm{~d}, 2 \mathrm{H}, J 7.1 \mathrm{~Hz}, \mathrm{Ar}-\mathrm{H}) .{ }^{13} \mathrm{C} \mathrm{NMR}\left(75 \mathrm{MHz}, \mathrm{CDCl}_{3}\right) \delta_{\mathrm{C}}: 13.7,21.5,50.9,54.8,60.0,89.7$, $112.3,127.5,128.5,128.7,129.0,129.5,133.3,134.0,136.2,141.3,144.8,156.9,163.2,192.3$. Anal. Calcd for $\mathrm{C}_{28} \mathrm{H}_{26} \mathrm{O}_{6} \mathrm{~S}: \mathrm{C}, 68.55$. H, 5.34\% Found C, 68.67; H, 5.25\%.

\section{trans-Ethyl5-benzoyl-4-p-tolyl-2-((p-methylphenylsulfonyl)methyl)-4,5-dihydrofuran-3-ca-} rboxylate (10b). Pale yellow solid; yield 68\%, mp 112-113 ${ }^{\circ} \mathrm{C} .{ }^{1} \mathrm{H}$ NMR $\left(300 \mathrm{MHz}, \mathrm{CDCl}_{3}\right) \delta_{\mathrm{H}}$ : 0.99 (t, 3H, J $\left.7.2 \mathrm{~Hz}, \mathrm{CH}_{3}\right), 2.36\left(\mathrm{~s}, 3 \mathrm{H}, \mathrm{CH}_{3}\right), 2.41\left(\mathrm{~s}, 3 \mathrm{H}, \mathrm{CH}_{3}\right.$ ) $3.81-3.91\left(\mathrm{~m}, 2 \mathrm{H}, \mathrm{CH}_{2}\right), 4.45$ $(\mathrm{d}, 1 \mathrm{H}, J 5.1 \mathrm{~Hz}), 4.55(\mathrm{~d}, 1 \mathrm{H}, J 13.8 \mathrm{~Hz}), 4.99(\mathrm{~d}, 1 \mathrm{H}, J 13.8 \mathrm{~Hz}),, 5.71(\mathrm{~d} .1 \mathrm{H}, J 5.1 \mathrm{~Hz}), 7.13$ (d, 2H, J 8.1 Hz, Ar-H), $7.16(\mathrm{~d}, 2 \mathrm{H}, J 8.1 \mathrm{~Hz}$, Ar-H), 7.26-7.30 (m, 2H, Ar-H) 7.45 (t, 2H, J 7.8 $\mathrm{Hz}, \mathrm{Ar}-\mathrm{H}), 7.61$ (t, 1H, J 7.8 Hz, Ar-H), 7.82 (d, 2H, J 7.2 Hz, Ar-H), 7.87 (d, 2H, J 8.1 Hz, ArH). ${ }^{13} \mathrm{C}$ NMR $\left(75 \mathrm{MHz}, \mathrm{CDCl}_{3}\right) \delta_{\mathrm{C}}: 13.7,21.0,21.5,50.6,54.9,60.0,89.8,112.4,127.4,128.1$, 128.5, 129.0, 129.4, 129.9, 133.3, 133.9, 136.3, 137.2, 138.3, 144.7, 156.8, 163.2, 192.4. Anal. Calcd for $\mathrm{C}_{29} \mathrm{H}_{28} \mathrm{O}_{6} \mathrm{~S}: \mathrm{C}, 69.03 ; \mathrm{H}, 5.59 \%$ Found $\mathrm{C}, 69.13 ; \mathrm{H}, 5.65 \%$.

trans-Ethyl5-benzoyl-4-(4-chlorophenyl)-2-((p-methylphenylsulfonyl)methyl)-4,5-dihydrofuran-3-carboxylate (10c). Pale yellow solid; yield 72\%, mp 148-149 ${ }^{\circ} \mathrm{C}{ }^{1} \mathrm{H}$ NMR $(300 \mathrm{MHz}$, $\left.\mathrm{CDCl}_{3}\right) \delta_{\mathrm{H}}: 1.01\left(\mathrm{t}, 3 \mathrm{H}, J 7.2 \mathrm{~Hz}, \mathrm{CH}_{3}\right), 2.43(\mathrm{~s}, 3 \mathrm{H}, \mathrm{CH} 3), 3.82-3.95\left(\mathrm{~m}, 2 \mathrm{H}, \mathrm{CH}_{2}\right), 4.50(\mathrm{~d}, 1 \mathrm{H}$, $J 5.1 \mathrm{~Hz}), 4.54(\mathrm{~d}, 1 \mathrm{H}, J 13.8 \mathrm{~Hz}), 5.02(\mathrm{~d}, 1 \mathrm{H}, J 13.8 \mathrm{~Hz}),, 5.68(\mathrm{~d} .1 \mathrm{H}, J 5.1 \mathrm{~Hz}), 7.26-7.35$ (m, 5H, Ar-H), 7.46 (t, 2H, J 7.1 Hz, Ar-H), 7.63 (t, 2H, J 7.1 Hz, Ar-H), $7.80-7.87$ (m, 4H, Ar-H). ${ }^{13} \mathrm{C}$ NMR $\left(75 \mathrm{MHz}, \mathrm{CDCl}_{3}\right) \delta_{\mathrm{C}}: 13.8,21.6,50.2,54.8,60.2,89.5,112.1,126.6,127.5$, 128.4, 128.8, 129.0, 129.0, 129.6, 133.4, 134.1, 136.4, 139.9, 144.9, 157.2, 163.9, 192.6. Anal. Calcd for : $\mathrm{C}_{28} \mathrm{H}_{25} \mathrm{ClO}_{6} \mathrm{~S}$ : C, 64.06; H, 4.80\% Found C, 63.95; H, 4.87\%.

trans-Ethyl5-benzoyl-4-(4-nitrophenyl)-2-((p-methylphenylsulfonyl)methyl)-4,5-dihydrofuran-3-carboxylate (10d). Pale yellow solid; yield 71\%, mp 137-138 ${ }^{\circ} \mathrm{C} .{ }^{1} \mathrm{H}$ NMR $(300 \mathrm{MHz}$, $\left.\mathrm{CDCl}_{3}\right) \delta_{\mathrm{H}}: 1.04\left(\mathrm{t}, 3 \mathrm{H}, J 7.2 \mathrm{~Hz}, \mathrm{CH}_{3}\right), 2.47$ (s, 3H, CH3), 3.90- 3.99 (m, 2H, CH ), 4.52 (d, 1H, $J 13.8 \mathrm{~Hz}), 4.80(\mathrm{~d}, 1 \mathrm{H}, J 5.1 \mathrm{~Hz}), 5.07(\mathrm{~d}, 1 \mathrm{H}, J 13.8 \mathrm{~Hz}),, 5.70(\mathrm{~d} .1 \mathrm{H}, J 5.1 \mathrm{~Hz}), 7.33-7.53$ (m, 6H, Ar-H), 7.67 (t, 1H, J 7.1 Hz, Ar-H), 7.85 - 7.88 (m, 4H, Ar-H), 8.26 (d, 2H, J 7.1 Hz ArH). ${ }^{13} \mathrm{C}$ NMR $\left(75 \mathrm{MHz}, \mathrm{CDCl}_{3}\right) \delta_{\mathrm{C}}: 13.8,21.6,26.8,54.8,60.4,89.1,111.7,124.1,128.3,128.6$, 128.9, 129.1, 129.7, 133.2, 134.3, 136.4, 145.1, 147.4, 148.7, 157.7, 162.8, 191.6 Anal. Calcd for $\mathrm{C}_{28} \mathrm{H}_{25} \mathrm{NO}_{8} \mathrm{~S}: \mathrm{C}, 62.79 ; \mathrm{H}, 4.71 ; \mathrm{N}, 2.62 \%$ Found C, 62.88; H, 4.61; N 2.71\%.

trans-Ethyl5-benzoyl-4-(2-methoxyphenyl)-2-((p-methylphenylsulfonyl)methyl)-4,5-dihydrofuran-3-carboxylate (10e). Pale yellow solid; yield 74\%, mp 115-116 ${ }^{\circ} \mathrm{C}$. ${ }^{1} \mathrm{H}$ NMR $(300$ $\left.\mathrm{MHz}, \mathrm{CDCl}_{3}\right) \delta_{\mathrm{H}}: 0.99\left(\mathrm{t}, 3 \mathrm{H}, J 7.2 \mathrm{~Hz}, \mathrm{CH}_{3}\right), 2.42\left(\mathrm{~s}, 3 \mathrm{H}, \mathrm{CH}_{3}\right), 3.61\left(\mathrm{~s}, 3 \mathrm{H}, \mathrm{OCH}_{3}\right), 3.84-3.97$ 
$\left(\mathrm{m}, 2 \mathrm{H}, \mathrm{CH}_{2}\right), 4.58(\mathrm{~d}, 1 \mathrm{H}, J 13.8 \mathrm{~Hz}), 4.94(\mathrm{~d}, 1 \mathrm{H}, J 5.1 \mathrm{~Hz}), 4.97$ (d, 1H, J $\left.13.8 \mathrm{~Hz},\right), 5.67$ (d. $1 \mathrm{H}, J 5.1 \mathrm{~Hz}), 6.85-7.61(\mathrm{~m}, 9 \mathrm{H}, \mathrm{Ar}-\mathrm{H}), 7.83-7.89(\mathrm{~m}, 4 \mathrm{H}, \mathrm{Ar}-\mathrm{H}) .{ }^{13} \mathrm{C} \mathrm{NMR}(75 \mathrm{MHz}$, $\left.\mathrm{CDCl}_{3}\right) \delta_{\mathrm{C}}: 13.8,21.5,44.7,54.8,55.0,59.9,89.2,111.0,120.7,126.2,127.3,128.5$, 128.8,129.0, 129.2, 129.5, 133.6, 134.0, 135.8, 136.2, 140.3, 144.7, 156.5, 163.5, 192.8. Anal. Calcd for : $\mathrm{C}_{29} \mathrm{H}_{28} \mathrm{O}_{7} \mathrm{~S}: \mathrm{C}, 66.91 ; \mathrm{H}, 5.42 \%$ Found C, 69.98; H, 5.33\%.

trans-Ethyl5-benzoyl-4-(2-bromophenyl)-2-((p-methylphenylsulfonyl)methyl)-4,5-dihydrofuran-3-carboxylate (10f). Pale yellow solid; yield 69\%, mp 132-133 ${ }^{\circ} \mathrm{C} .{ }^{1} \mathrm{H} \mathrm{NMR}(300 \mathrm{MHz}$, $\left.\mathrm{CDCl}_{3}\right) \delta_{\mathrm{H}}: 0.99\left(\mathrm{t}, 3 \mathrm{H}, J 7.2 \mathrm{~Hz}, \mathrm{CH}_{3}\right), 2.42\left(\mathrm{~s}, 3 \mathrm{H}, \mathrm{CH}_{3}\right), 3.84-3.97\left(\mathrm{~m}, 2 \mathrm{H}, \mathrm{CH}_{2}\right), 4.54(\mathrm{~d}, 1 \mathrm{H}$, $J 13.8 \mathrm{~Hz}), 5.01(\mathrm{~d}, 1 \mathrm{H}, J 13.8 \mathrm{~Hz}), 5.22(\mathrm{~d}, 1 \mathrm{H}, J 5.1 \mathrm{~Hz}), 5.68(\mathrm{~d}, 1 \mathrm{H}, J 5.1 \mathrm{~Hz}), 7.27-7.64$ (m, 9H, Ar-H), $7.83-7.87(\mathrm{~m}, 4 \mathrm{H}, \mathrm{Ar}-\mathrm{H}) .{ }^{13} \mathrm{C} \mathrm{NMR}\left(75 \mathrm{MHz}, \mathrm{CDCl}_{3}\right) \delta_{\mathrm{C}}: 13.8,21.6,49.1,54.7$, 60.1, 88.8, 112.2, 123.8, 126.3, 127.4, 127.9, 128.5, 128.8, 129.0, 129.6, 132.8, 133.6, 134.0, 136.4, 140.4, 144.9, 157.5, 163.2, 191.6 Anal. Calcd for $\mathrm{C}_{28} \mathrm{H}_{25} \mathrm{BrO}_{6} \mathrm{~S}: \mathrm{C}, 59.06 ; \mathrm{H}, 4.42 \%$ Found C, 59.18; H, 4.52\%.

trans-Ethyl5-benzoyl-4-(3-fluorophenyl)-2-((p-methylphenylsulfonyl)methyl)-4,5-dihydrofuran-3-carboxylate (10g). Pale yellow solid; yield 64\%, mp 118-119 ${ }^{\circ} \mathrm{C} .{ }^{1} \mathrm{H} \mathrm{NMR}(300 \mathrm{MHz}$, $\left.\mathrm{CDCl}_{3}\right) \delta_{\mathrm{H}}: 0.99\left(\mathrm{t}, 3 \mathrm{H}, J 7.2 \mathrm{~Hz}, \mathrm{CH}_{3}\right), 2.41\left(\mathrm{~s}, 3 \mathrm{H}, \mathrm{CH}_{3}\right), 3.79-3.97\left(\mathrm{~m}, 2 \mathrm{H}, \mathrm{CH}_{2}\right), 4.53(\mathrm{~d}, 1 \mathrm{H}$, $J 13.8 \mathrm{~Hz}), 4.56(\mathrm{~d}, 1 \mathrm{H}, J 5.1 \mathrm{~Hz}), 5.02(\mathrm{~d}, 1 \mathrm{H}, J 13.8 \mathrm{~Hz}), 5.68(\mathrm{~d}, 1 \mathrm{H}, J 5.1 \mathrm{~Hz}), 6.88-7.05$ (m, 2H, Ar-H), $7.28-7.38(\mathrm{~m}, 4 \mathrm{H}, \mathrm{Ar}-\mathrm{H}), 7.46(\mathrm{t}, 2 \mathrm{H}, J 8.1 \mathrm{~Hz}, \mathrm{Ar}-\mathrm{H}), 7.62(\mathrm{t}, 1 \mathrm{H}, J 8.1 \mathrm{~Hz}$, $\mathrm{Ar}-\mathrm{H}), 7.83$ (d, 2H, J 8.1 Hz, Ar-H), $7.86(\mathrm{~d}, 2 \mathrm{H}, J 8.1 \mathrm{~Hz}, \mathrm{Ar}-\mathrm{H}) .{ }^{13} \mathrm{C}$ NMR $\left(75 \mathrm{MHz}, \mathrm{CDCl}_{3}\right)$ $\delta_{\mathrm{C}}: 13.8,21.6,50.3,54.8,60.2,89.4,111.9,123.3,126.4,127.6,128.2,128.5,128.8,129.1$, 129.6, 130.0, 130.4, 133.3, 134.2, 136.5, 145.0, 157.3, 163.0, 192.1. Anal. Calcd for: $\mathrm{C}_{28} \mathrm{H}_{25} \mathrm{FO}_{6} \mathrm{~S}: \mathrm{C}, 66.13 ; \mathrm{H}, 4.95 \%$ Found C, 66.06; H, 5.05\%.

trans-Ethyl5-benzoyl-4-(2,4-dichlorophenyl)-2-((p-methylphenylsulfonyl)methyl)-4,5-dihydrofuran-3-carboxylate (10h). Pale yellow solid; yield 75\%, mp 123-124 ${ }^{\circ} \mathrm{C} .{ }^{1} \mathrm{H}$ NMR $(300$ $\left.\mathrm{MHz}_{\mathrm{CDCl}}\right)_{3} \delta_{\mathrm{H}}: 1.06\left(\mathrm{t}, 3 \mathrm{H}, J 7.2 \mathrm{~Hz}, \mathrm{CH}_{3}\right), 2.46(\mathrm{~s}, 3 \mathrm{H}, \mathrm{CH} 3), 3.91-3.98\left(\mathrm{~m}, 2 \mathrm{H}, \mathrm{CH}_{2}\right), 4.52$ $(\mathrm{d}, 1 \mathrm{H}, J 13.8 \mathrm{~Hz}), 5.04(\mathrm{~d}, 1 \mathrm{H}, J 13.8 \mathrm{~Hz}), 5.22(\mathrm{~d}, 1 \mathrm{H}, J 5.1 \mathrm{~Hz}), 5.67$ (d, 1H, J 5.1 Hz), 7.33 7.41 (m, 5H, Ar-H), 7.49 (t, 2H, J 8.1 Hz, Ar-H), 7.65 (t, 1H, J 8.1 Hz, Ar-H), 7.87 (d, 2H, J 8.1 $\mathrm{Hz}, \mathrm{Ar}-\mathrm{H}), 7.88$ (d, $2 \mathrm{H}, J 8.1 \mathrm{~Hz}, \mathrm{Ar}-\mathrm{H}) .{ }^{13} \mathrm{C} \mathrm{NMR}\left(75 \mathrm{MHz}, \mathrm{CDCl}_{3}\right) \delta_{\mathrm{C}}: 13.8,21.6,49.2,54.7$, 60.3, 88.4, 111.7, 123.2, 127.9, 128.3, 128.8, 129.1, 129.3, 129.6, 130.0, 133.9, 134.1, 136.4, 145.0, 157.7, 163.0, 191.4. Anal. Calcd for $\mathrm{C}_{28} \mathrm{H}_{24} \mathrm{Cl}_{2} \mathrm{O}_{6} \mathrm{~S}: \mathrm{C}, 60.11 ; \mathrm{H}, 4.32 \%$ Found C, 60.23; $\mathrm{H}, 4.40 \%$.

trans-Ethyl5-benzoyl-2-((4-chlorophenylsulfonyl)methyl)-4-(4-methylphenyl)-4,5-dihydrofuran-3-carboxylate (10i). Pale yellow solid; yield 67\%, mp 104-105 ${ }^{\circ} \mathrm{C} .{ }^{1} \mathrm{H} \mathrm{NMR}(300 \mathrm{MHz}$, $\left.\mathrm{CDCl}_{3}\right) \delta_{\mathrm{H}}: 0.99\left(\mathrm{t}, 3 \mathrm{H}, J 7.2 \mathrm{~Hz}, \mathrm{CH}_{3}\right), 2.37\left(\mathrm{~s}, 3 \mathrm{H}, \mathrm{CH}_{3}\right), 3.81-3.89\left(\mathrm{~m}, 2 \mathrm{H}, \mathrm{CH}_{2}\right), 4.44(\mathrm{~d}, 1 \mathrm{H}$, $J 5.1 \mathrm{~Hz}), 4.65$ (d, 1H, J 13.8 Hz), $4.95(\mathrm{~d}, 1 \mathrm{H}, J 13.8 \mathrm{~Hz}), 5.74$ (d, 1H, J 5.1 Hz), 7.09 (d, 2H, J $=8.1 \mathrm{~Hz}, \mathrm{Ar}-\mathrm{H}), 7.16(\mathrm{~d}, 2 \mathrm{H}, J 8.1 \mathrm{~Hz}, \mathrm{Ar}-\mathrm{H}), 7.44-7.48(\mathrm{~m}, 4 \mathrm{H}, \mathrm{Ar}-\mathrm{H}), 7.62(\mathrm{t}, 1 \mathrm{H}, J$ 7.2, Ar$\mathrm{H}), 7.81(\mathrm{~d}, 2 \mathrm{H}, J 8.1 \mathrm{~Hz}, \mathrm{Ar}-\mathrm{H}), 7.94(\mathrm{~d}, 2 \mathrm{H}, J 8.1 \mathrm{~Hz}, \mathrm{Ar}-\mathrm{H}) .{ }^{13} \mathrm{C} \mathrm{NMR}\left(75 \mathrm{MHz}, \mathrm{CDCl}_{3}\right) \delta_{\mathrm{C}}$ : 13.8, 21.1, 50.6, 54.9, 60.2, 89.9, 112.8, 127.5, 128.9, 129.1, 129.2, 129.6, 130.3, 133.5, 134.1, 137.4, 137.7, 138.3, 140.7, 156.5, 163.3, 192.4. Anal. Calcd for : $\mathrm{C}_{28} \mathrm{H}_{25} \mathrm{ClO}_{6} \mathrm{~S}: \mathrm{C}, 64.06$; $\mathrm{H}$, $4.80 \%$ Found C, 64.17; H, 4.68\%. 
trans-Ethyl5-benzoyl-2-((4-chlorophenylsulfonyl)methyl)-4-(4-isopropylphenyl)-4,5-dihydrofuran-3-carboxylate (10j). Pale yellow solid; yield 60\%; mp 127-128 ${ }^{\circ} \mathrm{C}$. ${ }^{1} \mathrm{H}$ NMR (300 $\left.\mathrm{MHz}, \mathrm{CDCl}_{3}\right) \delta_{\mathrm{H}}: 0.98\left(\mathrm{t}, 3 \mathrm{H}, J 7.2 \mathrm{~Hz}, \mathrm{CH}_{3}\right), 1.26\left(\mathrm{~s}, 3 \mathrm{H}, \mathrm{CH}_{3}\right), 1.28\left(\mathrm{~s}, 3 \mathrm{H}, \mathrm{CH}_{3}\right), 2.91-2.96$ $(\mathrm{m}, 1 \mathrm{H}, \mathrm{CH}) 3.83-3.90\left(\mathrm{~m}, 2 \mathrm{H}, \mathrm{CH}_{2}\right), 4.47(\mathrm{~d}, 1 \mathrm{H}, J 5.1 \mathrm{~Hz}), 4.65(\mathrm{~d}, 1 \mathrm{H}, J 13.8 \mathrm{~Hz}), 4.95(\mathrm{~d}$, $1 \mathrm{H}, J 13.8 \mathrm{~Hz}), 5.76(\mathrm{~d}, 1 \mathrm{H}, J 5.1 \mathrm{~Hz}), 7.12(\mathrm{~d}, 2 \mathrm{H}, J 8.1 \mathrm{~Hz}, \mathrm{Ar}-\mathrm{H}) 7.22(\mathrm{~d}, 2 \mathrm{H}, J 8.1 \mathrm{~Hz}, \mathrm{Ar}-\mathrm{H})$, 7.42 - 7.48 (m, 4H, Ar-H), 7.59 - 7.62 (m, 1H, Ar-H), 7.83 (d, 2H, J 8.1 Hz, Ar-H), 7.93 (d, 2H, $J$ 8.1 Hz, Ar-H). ${ }^{13} \mathrm{C}$ NMR $\left(75 \mathrm{MHz}, \mathrm{CDCl}_{3}\right) \delta_{\mathrm{C}}: 13.8,23.9,26.9,33.8,50.5,54.8,60.2,89.8$, $112.9,126.9$, 127.5, 128.5, 128.9, 129.1, 130.2, 133.3, 134.1, 137.4, 138.1, 140.6, 148.4, 156.4, 163.3, 192.0. Anal. Calcd for $\mathrm{C}_{30} \mathrm{H}_{29} \mathrm{ClO}_{6} \mathrm{~S}: \mathrm{C}, 65.15 ; \mathrm{H}, 5.29 \%$ Found C, 65.08; H, 5.38\%

trans-Ethyl5-benzoyl-4-(4-chlorophenyl)-2-((4-chlorophenylsulfonyl)methyl)-4,5-dihydrofuran-3-carboxylate (10k). Pale yellow solid; yield 67\%, mp 139-140 ${ }^{\circ} \mathrm{C}$. ${ }^{1} \mathrm{H}$ NMR $(300 \mathrm{MHz}$, $\left.\mathrm{CDCl}_{3}\right) \delta_{\mathrm{H}}: 1.00\left(\mathrm{t}, 3 \mathrm{H}, J 7.2 \mathrm{~Hz}, \mathrm{CH}_{3}\right), 3.84-3.94\left(\mathrm{~m}, 2 \mathrm{H}, \mathrm{CH}_{2}\right), 4.52(\mathrm{~d}, 1 \mathrm{H}, J 5.1 \mathrm{~Hz}), 4.63(\mathrm{~d}$, $1 \mathrm{H}, J 13.8 \mathrm{~Hz}), 4.95(\mathrm{~d}, 1 \mathrm{H}, J 13.8 \mathrm{~Hz}), 5.69$ (d, 1H, J 5.1 Hz), 7.16 (d, 2H, J 8.1 Hz, Ar-H), 7.34 (d, 2H, J 8.1 Hz, Ar-H), $7.44-7.49$ (m, 4H, Ar-H), 7.63 (t, 1H, J $7.1 \mathrm{~Hz}$, Ar-H), 7.81 (d, $2 \mathrm{H}, J 8.1 \mathrm{~Hz}, \mathrm{Ar}-\mathrm{H}), 7.91(\mathrm{~d}, 2 \mathrm{H}, J 8.1 \mathrm{~Hz}, \mathrm{Ar}-\mathrm{H}) .{ }^{13} \mathrm{C} \mathrm{NMR}\left(75 \mathrm{MHz}, \mathrm{CDCl}_{3}\right) \delta_{\mathrm{C}}: 13.8,50.2$, $54.8,60.3,89.5,112.4,126.3,127.4,128.9,129.1,129.3,130.1,133.2,133.6,134.3,137.5$, 139.8, 140.7, 156.9, 163.1, 191.9. Anal. Calcd for $\mathrm{C}_{27} \mathrm{H}_{22} \mathrm{Cl}_{2} \mathrm{O}_{6} \mathrm{~S}$ : C, 59.46; H, 4.07\% Found C, $59.52 ; \mathrm{H}, 4.18 \%$.

trans-Ethyl5-benzoyl-2-((4-chlorophenylsulfonyl)methyl)-4-(4-methoxyphenyl)-4,5-dihydrofuran-3-carboxylate (10l). Pale yellow solid; yield 70\%; mp 120-121 ${ }^{\circ} \mathrm{C} .{ }^{1} \mathrm{H}$ NMR $(300 \mathrm{MHz}$, $\left.\mathrm{CDCl}_{3}\right) \delta_{\mathrm{H}}: 0.99\left(\mathrm{t}, 3 \mathrm{H}, J 7.2 \mathrm{~Hz}, \mathrm{CH}_{3}\right), 3.80\left(\mathrm{~s}, 3 \mathrm{H}, \mathrm{OCH}_{3}\right), 3.82-3.94\left(\mathrm{~m}, 2 \mathrm{H}, \mathrm{CH}_{2}\right), 4.42(\mathrm{~d}, 1 \mathrm{H}$, $J 5.1 \mathrm{~Hz}), 4.64$ (d, 1H, J 13.8 Hz), $4.96(\mathrm{~d}, 1 \mathrm{H}, J 13.8 \mathrm{~Hz}), 5.73(\mathrm{~d}, 1 \mathrm{H}, J 5.1 \mathrm{~Hz}), 6.89$ (d, 2H, J $8.1 \mathrm{~Hz}, \mathrm{Ar}-\mathrm{H}), 7.12$ (d, 2H, J 8.1 Hz, Ar-H), $7.43-7.48$ (m, 4H, Ar-H), 7.62 (t, 1H, J 7.1 Hz, $\mathrm{Ar}-\mathrm{H}), 7.81$ (d, 2H, J 8.1 Hz, Ar-H), 7.94 (d, 2H, $J 8.1 \mathrm{~Hz}, \mathrm{Ar}-\mathrm{H}) .{ }^{13} \mathrm{C}$ NMR $\left(75 \mathrm{MHz}, \mathrm{CDCl}_{3}\right)$ $\delta_{\mathrm{C}}: 13.8,50.7,54.8,54.3,60.2,89.9,112.8,126.4,127.8,128.7,128.9,129.1,129.2,130.2$, 133.3, 134.2, 137.5, 140.7, 156.4, 159.1, 163.3, 192.4. Anal. Calcd for $\mathrm{C}_{28} \mathrm{H}_{25} \mathrm{ClO}_{7} \mathrm{~S}: \mathrm{C}, 62.16$; H, 4.66\% Found C, 62.08; H, 4.55\%.

trans-Ethyl5-benzoyl-4-(2-bromophenyl)-2-((4-chlorophenylsulfonyl)methyl)-4,5-dihydrofuran-3-carboxylate (10m). Pale yellow solid; yield 70\%; mp 128-129 ${ }^{\circ} \mathrm{C} .{ }^{1} \mathrm{H}$ NMR $(300 \mathrm{MHz}$, $\left.\mathrm{CDCl}_{3}\right) \delta_{\mathrm{H}}: 0.99\left(\mathrm{t}, 3 \mathrm{H}, J 7.2 \mathrm{~Hz}, \mathrm{CH}_{3}\right), 3.84-3.94\left(\mathrm{~m}, 2 \mathrm{H}, \mathrm{CH}_{2}\right), 4.66(\mathrm{~d}, 1 \mathrm{H}, J 13.8 \mathrm{~Hz}), 4.93$ (d, 1H, J 13.8 Hz), 5.21 (d, 1H, J 5.1 Hz), 5.69 (d, 1H, J 5.1 Hz), 7.27 - 7.64 (m, 9H, Ar-H), 7.83 - $7.87(\mathrm{~m}, 4 \mathrm{H}, \mathrm{Ar}-\mathrm{H}) .{ }^{13} \mathrm{C}$ NMR $\left(75 \mathrm{MHz} \mathrm{CDCl}_{3}\right) \delta_{\mathrm{C}}: 13.7,48.9,54.6,60.3,88.7,112.4$, 123.8, 126.4, 127.6, 128.2, 128.5, 128.8, 129.1, 130.1, 132.9, 133.5, 134.1, 136.2, 137.5, 140.7, 157.2, 163.2, 191.4. Anal. Calcd for $\mathrm{C}_{27} \mathrm{H}_{22} \mathrm{BrClO}_{6} \mathrm{~S}: \mathrm{C}, 54.98 ; \mathrm{H}, 3.76 \%$ Found C, 55.07; H, $3.65 \%$.

trans-Ethyl5-benzoyl-4-(3-bromophenyl)-2-((4-chlorophenylsulfonyl)methyl)-4,5-dihydrofuran-3-carboxylate (10n). Pale yellow solid; yield 70\%, mp 139-140 ${ }^{\circ} \mathrm{C}$. ${ }^{1} \mathrm{H}$ NMR (300 MHz, $\left.\mathrm{CDCl}_{3}\right) \delta_{\mathrm{H}}: 0.99\left(\mathrm{t}, 3 \mathrm{H}, J 7.2 \mathrm{~Hz}, \mathrm{CH}_{3}\right), 3.78-3.96\left(\mathrm{~m}, 2 \mathrm{H}, \mathrm{CH}_{2}\right), 4.53(\mathrm{~d}, 1 \mathrm{H}, J 5.1 \mathrm{~Hz}), 4.62(\mathrm{~d}$, $1 \mathrm{H}, J 13.8 \mathrm{~Hz}), 4.92(\mathrm{~d}, 1 \mathrm{H}, J 13.8 \mathrm{~Hz}), 5.72(\mathrm{~d}, 1 \mathrm{H}, J 5.1 \mathrm{~Hz}), 7.15-7.26(\mathrm{~m}, 2 \mathrm{H}, \mathrm{Ar}-\mathrm{H}), 7.41-$ 7.49 (m, 5H, Ar-H), 7.63 (t, 1H, J $8.1 \mathrm{~Hz}$, Ar-H), 7.82 (t, 1H, J 8.1 Hz, Ar-H), 7.90 (d, 2H, J 8.1 
$\mathrm{Hz}, \mathrm{Ar}-\mathrm{H}), 7.86\left(\mathrm{~d}, 2 \mathrm{H}, J\right.$ 8.1 Hz, Ar-H). ${ }^{13} \mathrm{C} \mathrm{NMR}\left(75 \mathrm{MHz}, \mathrm{CDCl}_{3}\right) \delta_{\mathrm{C}}: 13.8,50.1,54.7,60.3$, $89.3,112.2,122.9,126.3,128.9,129.1,129.3,130.1,130.5,130.7,130.9,133.2,134.3$, 137.4,140.7, 143.5, 156.9, 162.9, 191.8. Anal. Calcd for $\mathrm{C}_{27} \mathrm{H}_{22} \mathrm{BrClO}_{6} \mathrm{~S}: \mathrm{C}, 54.98 ; \mathrm{H}, 3.76 \%$ Found C, 55.05; H, 3.85\%.

trans-Ethyl5-benzoyl-2-((4-chlorophenylsulfonyl)methyl)-4-(3-fluorophenyl)-4,5-dihydrofuran-3-carboxylate (10o). Pale yellow solid; yield 70\%, mp 115-116 ${ }^{\circ} \mathrm{C}$. ${ }^{1} \mathrm{H} \mathrm{NMR}(300 \mathrm{MHz}$, $\left.\mathrm{CDCl}_{3}\right) \delta_{\mathrm{H}}: 0.98\left(\mathrm{t}, 3 \mathrm{H}, J 7.2 \mathrm{~Hz}, \mathrm{CH}_{3}\right), 3.81-3.96\left(\mathrm{~m}, 2 \mathrm{H}, \mathrm{CH}_{2}\right), 4.55(\mathrm{~d}, 1 \mathrm{H}, J 5.1 \mathrm{~Hz}), 4.64(\mathrm{~d}$, $1 \mathrm{H}, J 13.8 \mathrm{~Hz}), 4.93(\mathrm{~d}, 1 \mathrm{H}, J 13.8 \mathrm{~Hz}), 5.71(\mathrm{~d}, 1 \mathrm{H}, J 5.1 \mathrm{~Hz}), 6.91-7.02$ (m, 3H, Ar-H), $7.26-$ $7.65(\mathrm{~m}, 8 \mathrm{H}, \mathrm{Ar}-\mathrm{H}), 7.81(\mathrm{t}, 1 \mathrm{H}, J 8.1 \mathrm{~Hz}, \mathrm{Ar}-\mathrm{H}), 7.91\left(\mathrm{t}, 1 \mathrm{H}, J 8.1 \mathrm{~Hz}\right.$, Ar-H) ${ }^{13} \mathrm{C}$ NMR $(75$ $\left.\mathrm{MHz}, \mathrm{CDCl}_{3}\right) \delta_{\mathrm{C}}: 13.8,50.3,54.7,60.3,89.4,112.4,123.2,123.5,126.6,127.4,128.9,129.1$, $129.3,130.1,130.9,133.2,134.3,137.4,140.7,143.8,156.9,162.7,191.9$. Anal. Calcd for $\mathrm{C}_{27} \mathrm{H}_{22} \mathrm{ClFO}_{6} \mathrm{~S}: \mathrm{C}, 61.30 ; \mathrm{H}, 4.19 \%$ Found C, 61.22; H, $4.30 \%$.

trans-Ethyl5-benzoyl-2-((4-chlorophenylsulfonyl)methyl)-4-(2,4-dichlorophenyl)-4,5-dihydrofuran-3-carboxylate (10p). Pale yellow solid; yield 70\%, mp 139-140 ${ }^{\circ} \mathrm{C} .{ }^{1} \mathrm{H}$ NMR (300 $\left.\mathrm{MHz}, \mathrm{CDCl}_{3}\right) \delta_{\mathrm{H}}: 1.03\left(\mathrm{t}, 3 \mathrm{H}, J 7.2 \mathrm{~Hz}, \mathrm{CH}_{3}\right), 3.85-3.95\left(\mathrm{~m}, 2 \mathrm{H}, \mathrm{CH}_{2}\right), 4.62(\mathrm{~d}, 1 \mathrm{H}, J 13.8 \mathrm{~Hz})$, $4.94(\mathrm{~d}, 1 \mathrm{H}, J 13.8 \mathrm{~Hz}), 5.20$ (d, 1H, J 5.1 Hz), 5.66 (d, 1H, J 5.1 Hz), 7.22 - 7.62 (m, 7H, ArH), $7.83(\mathrm{~d}, 2 \mathrm{H}, J 8.1 \mathrm{~Hz}, \mathrm{Ar}-\mathrm{H}), 7.91(\mathrm{~d}, 2 \mathrm{H}, J 8.1 \mathrm{~Hz}, \mathrm{Ar}-\mathrm{H}), 8.12(\mathrm{~d}, 1 \mathrm{H}, J 7.2 \mathrm{~Hz}, \mathrm{Ar}-\mathrm{H}) .{ }^{13} \mathrm{C}$ NMR $\left(75 \mathrm{MHz}, \mathrm{CDCl}_{3}\right) \delta_{\mathrm{C}}: 13.8,49.2,54.7,60.3,88.4,111.7,123.2,127.9,128.3,128.8,129.1$, 129.3, 129.6, 130.0, 133.9, 134.1, 136.4, 145.0, 157.7, 163.0, 191.4. Anal. Calcd for $\mathrm{C}_{27} \mathrm{H}_{21} \mathrm{Cl}_{3} \mathrm{O}_{6} \mathrm{~S}: \mathrm{C}, 55.92 ; \mathrm{H}, 3.65 \%$ Found C, 56.04; H, 3.73\%.

\section{Acknowledgements}

S.P. and A.I.M. gratefully acknowledge the Deanship of Scientific Research at King Saud University for funding through the research Grant RGP-VPP-026.

\section{References}

1. Ugi, I.; Domling, A.; Horl, W. Endeavour 1994, 18, 115. http://dx.doi.org/10.1016/S0160-9327(05)80086-9

2. Armstrong, R. M.; Combs, A. P.; Tempest, P. A.; Brown, S. D.; Keating, T. A. Acc. Chem. Res. 1996, 29, 123. http://dx.doi.org/10.1021/ar9502083

3. Dömling, A. Chem. Rev. 2006, 106, 17. http://dx.doi.org/10.1021/cr0505728

4. Soleimani, E.; Khodaei, M. M.; Batooie, N.; Baghbanzadeh, M. Green Chem. 2011, 13, 566. http://dx.doi.org/10.1039/c0gc00739k 
5. L. F. Tietze, Chem. Rev., 1996, 96, 115. http://dx.doi.org/10.1021/cr950027e

6. Nair, V.; Rajesh, C.; Vinod, A. U.; Bindu, S.; Sreekanth, A. R.; Mathen, J. S.; Balagopal, L. Acc. Chem. Res. 2003, 36, 899. http://dx.doi.org/10.1021/ar020258p

7. Ramon, D. J.; Yus, M. Angew. Chem., Int. Ed. 2005, 44, 1602. http://dx.doi.org/10.1002/anie.200460548

8. Nicolaou, K. C.; Edmonds, D. J.; Bulger. P. G. Angew. Chem., Int. Ed., 2006, 45, 7134. http://dx.doi.org/10.1002/anie.200601872

9. Grondal, C.; Jeanty, M.; Enders. D. Nat. Chem., 2010, 2, 167. http://dx.doi.org/10.1038/nchem.539

10. Danheiser, R. L.; Stoner, E. J.; Kojama, H.; Yamashita, D. S.; Klade, C. A. J. Am. Chem. Soc. 1989, 111, 4407.

http://dx.doi.org/10.1021/ja00194a040

11. Fraga, B. M. Nat. Prod. Rep. 1992, 9, 217. http://dx.doi.org/10.1039/np9920900217

12. Merrit, A. T.; Ley, S. V. Nat. Prod. Rep. 1992, 9, 243. http://dx.doi.org/10.1039/np9920900243

13. http://apps.who.int/medicinedocs/en/d/Jh2988e/14.2.html

14. Xiang, J.; Ipek, M.; Suri, V.; Tam, M.; Xing, Y.; Huang, N.; Zhang, Y.; Tobin, J.;

Mansour, T.S.; McKew, J. Bioorg. Med. Chem.2007, 15, 4396. http://dx.doi.org/10.1016/j.bmc.2007.04.035

15. Dominguez, J. N.; Leon, C.; Rodrigues, J.; Gamboa de Dominguez, N.; Gut, J.; Rosenthal, P. J. European J. Med. Chem. 2009, 44, 1457. http://dx.doi.org/10.1016/j.ejmech.2008.09.044

16. Pal, T. K.; Dey, T.; Chakrabarty, A.; Dey, D.; Ghosh, S.K.; Pathak, T. Bioorg. Med. Chem. Lett. 2010, 20, 3777. http://dx.doi.org/10.1016/j.bmcl.2010.04.056

17. Ayers, B.; Long, H.; Sim, E.; Smellie, I.A.; Wilkinson, B.L.; Fairbanks, A. J. Carb. Res., 2009,344, 739 . http://dx.doi.org/10.1016/j.carres.2009.02.006

18. Zheng, J.-C.; Zhu, Y.-C.; Sun, X.-L.; Tang, Y.; Dai, L.-X. J. Org. Chem. 2008, 73, 6909. http://dx.doi.org/10.1021/jo801135j

19. Payne, G. B. J. Org. Chem. 1967, 32, 3351. http://dx.doi.org/10.1021/jo01286a017

20. Caló, V. J. Org. Chem. 2003, 68, 4406. http://dx.doi.org/10.1021/jo026849a

21. Xing, C.; Zhu, S. J. Org. Chem. 2004, 69, 6486. http://dx.doi.org/10.1021/jo049317y 
22. Aso, M.; Ojida, A.; Yang, G.; Cha, O. J.; Osawa, E.; Kamematsu, K. J. Org. Chem. 1993, 58,3960 .

http://dx.doi.org/10.1021/jo00067a031

23. Antonioletti, R.; Bovicelli, P.; Malancona, S. Tetrahedron 2002, 58, 589. (d) Lee, Y. R.; Suk, J. Y. Tetrahedron 2002, 58, 2359.

24. Evans, D. A.; Sweeney, Z. K.; Rovis, T.; Tedrow, J. S. J. Am. Chem. Soc. 2001, 123, 12095. http://dx.doi.org/10.1021/ja011983i

25. Xie, J-W.; Li, P.; Wang, T.; Zhou, F-T. Tetrahedron Lett. 2011, 52, 2379. http://dx.doi.org/10.1016/j.tetlet.2011.02.093

26. (a) Lee, P. H.; Kim, J. S.; Kim, S. Tetrahedron Lett. 1993, 34, 7583.

27. Nakajima, T.; Segi, M.; Mituoka, T.; Fukute, Y.; Honda, M.; Naitou, K. Tetrahedron Lett. 1995, 36, 1667.

http://dx.doi.org/10.1016/0040-4039(95)00125-V

28. Yadav, V. K.; Balamurugan, R. Org. Lett. 2001, 3, 2717. http://dx.doi.org/10.1021/o10163169

29. Alonso, M. E.; Morales, A. J. Org. Chem. 1980, 45, 4530. (f) Melot, J. M.; Texier- Boullet, F.; Foucaud, A. Tetrahedron 1988, 44, 2215.

30. Chuang, C-P.; Chen, K-P.; Tetrahedron 2012, 68, 1401. http://dx.doi.org/10.1016/j.tet.2011.12.035

31. Ye, Y.; Wang, L.; Fan, R. J. Org. Chem. 2010, 75, 1760. http://dx.doi.org/10.1021/jo902553k

32. Fan, L-P.; Li, P.; Li, X-S.; Xu, D-C.; Ge, M-M.; Zhu, W-D.; Xie, J-W. J. Org. Chem. 2010, 75, 8716. http://dx.doi.org/10.1021/jo101935k

33. Rueping, M.; Parra, A.; Uria, U.; Besselièvre, F.; Merino. E. Org. Lett. 2010, 12, 5680. http://dx.doi.org/10.1021/ol102499r

34. For representative examples see: (a) Perumal S.; Menéndez, J. C. Targets in Heterocyclic Systems - Chemistry and Properties, volume 15. Eds. Orazio A. Attanasi and Domenico Spinelli, 2011, 402.

35. Devi Bala, B.; Michael Rajesh, M.; Perumal, S. Green Chem. 2012, 14, 2484. http://dx.doi.org/10.1039/c2gc35930h

36. Indumathi, S.; Perumal, S.; Anbananthan. N. Green Chem. 2012, 14, 3361. http://dx.doi.org/10.1039/c2gc36040c

37. Michael Rajesh, S.; Bala, B. D.; Perumal, S.; Menéndez, J. C. Green Chem. 2011, 13, 3248 .

http://dx.doi.org/10.1039/c1gc15794a

38. Indumathi, S.; Perumal, S.; Menendez, J.C. J. Org. Chem. 2010, 75, 472. http://dx.doi.org/10.1021/jo9021327 
39. Crystallographic data for the derivative 10n in this paper is deposited with the Cambridge Crystallographic Data Centre as supplementary publication number CCDC 912394. Copies of the data can be obtained, free of charge, on application to CCDC, 12 Union Road, Cambridge CB2 1EZ, UK [fax: +44 (0)1223 336033 or e-mail: deposit@ ccdc.cam.ac.uk] 